\title{
Formation of Young Star Clusters
}

\author{
Bruce Elmegreen \\ IBM Research Division, T.J. Watson Research Center, PO Box 218, \\ Yorktown Hts., NY, 10598, USA, bge@watson.ibm.com
}

\begin{abstract}
Turbulence, self-gravity, and cooling convert most of the interstellar medium into cloudy structures that form stars. Turbulence compresses the gas into clouds directly and it moves pre-existing clouds around passively when there are multiple phases of temperature. Self-gravity also partitions the gas into clouds, forming giant regular complexes in spiral arms and in resonance rings and contributing to the scale-free motions generated by turbulence. Dense clusters form in the most strongly self-gravitating cores of these clouds, often triggered by compression from local stars. Pre-star formation processes inside clusters are not well observed, but the high formation rates and high densities of pre-stellar objects, and their power law mass functions suggest that turbulence, self-gravity, and energy dissipation are involved there too.
\end{abstract}

\section{Many Scales of Star Formation}

Star formation has many scales. Giant star complexes extend for $\sim 500 \mathrm{pc}$ along spiral arms and disperse in the inter-arm regions. The clouds that form them are usually visible in HI surveys, and their cores are visible in CO surveys (Grabelsky et al. 1987). These clouds are mildly self-bound by gravity (Elmegreen \& Elmegreen 1987; Rand 1993), so they are like any other star-forming clouds: virialized, supersonically turbulent, and producing stars in perhaps several generations with an efficiency of $\sim 10 \%$. The star formation process is confined to the densest cores of these clouds, where gravity is strong and thermal pressure is weak. Between these extremes of scale, the gas temperature decreases and the molecular content increases, but the physical processes that cause stars to form in aggregates do not change much. These processes are a combination of multiscale and repetitive compressions from supersonic turbulence and self-gravity, energy dissipation through shocks and magnetic diffusion, and collapse from overwhelming gravitational forces. Some of the complexity of star-formation dynamics is shown in the simulations by Bate, Bonnell, \& Bromm (2003).

\section{Scale-dependent Morphologies}

Corresponding to the many scales of star formation, self-gravitating clouds have a wide range of masses, from $\sim 10^{7} \mathrm{M}_{\odot}$ to less than $1 \mathrm{M}_{\odot}$ in our Galaxy. What a cloud produces is called a star cluster only if its mass exceeds $\sim 100 \mathrm{M}_{\odot}$ (Lada \& Lada 2003). Other than this, there is no characteristic or dominant mass for clouds or clusters, only power law distributions, so most star-forming regions are similar except for size. Size determines velocity dispersion and density for a 
common background pressure, and density variations lead to important morphological differences through two dimensionless ratios: the dynamical time divided by the evolution time of stars, and the dynamical time divided by the shear time in the local galaxy. The largest clouds take a short time, in relative terms, to form most of their stars: just 1 or 2 dynamical times like nearly every other cloud. But these largest clouds take a long time, in absolute terms, to do this, $\sim 40 \mathrm{My}$ in the case of Gould's Belt, and by then the oldest populations have lost their most massive members to stellar evolution, making the complexes look relatively dull (Efremov 1995). The largest clouds are also the most severely affected by shear, making them look like flocculent spiral arms or spiral arm spurs (Kim \& Ostriker 2002). These morphological differences disguise the fact that the physical processes of star and cluster formation are very similar on all scales.

Galactic-scale stellar dynamical processes can lead to the collection of gas into spiral density waves and resonance rings. Then the largest clouds are somewhat uniformly distributed along the length of the stellar structure with a characteristic separation equal to $\sim 3$ times the arm or ring thickness. What happens here is that clouds form by asymmetric gravitational instabilities with a converging flow along the length of the structure. Typically shear and galactic tidal forces are low in these regions, allowing the clouds to form in gas that would otherwise be stable (Rand 1993; Elmegreen 1994).

\section{Power Spectra}

When there are no galactic-scale structures, the gas appears completely scalefree, as in the Large and Small Magellanic Clouds (Stanimirovic et al. 1999; Elmegreen, Kim, \& Staveley-Smith 2001). Power spectra of the emission or absorption from this gas have power laws with a slope similar to that for velocity power spectra in incompressible turbulence, namely $\sim-2.8$ in two-dimensions (Stützki et al. 1998; Dickey et al. 2001). Incompressible turbulence has the Kolmogorov spectrum with a slope of $-8 / 3$. Why the column density structure in a medium that is supersonically turbulent should have about the same power spectrum as the velocity structure in incompressible turbulence is somewhat of a mystery, unless it is partly coincidence. The power spectrum of turbulent velocities varies by only a small amount, from $-8 / 3$ to -3 (in $2 \mathrm{D}$ ), as the motion varies from incompressible to shock-dominated. Thus even the most extreme cloud formation scenarios, where all clouds are shock fronts, would have a power spectrum similar to incompressible turbulence. In addition, some of the gas structure could result from entrainment of many tiny clouds in the larger-scale turbulent velocity field (Goldman 2000). Entrainment means density is a passive scalar, and then density power spectra are the same as velocity power spectra. Third, expanding shells make dense gas, and these introduce a -3 component to the power spectrum because of their sharp edges. The result is a mixture of processes and innate power spectra. This is why widely diverse morphologies ranging from flocculent dust spirals in galactic nuclei (Elmegreen, Elmegreen, \& Eberwein 2002) to shells and holes in the LMC or SMC (Kim et al. 1999; Stanimirovic et al. 1999; Elmegreen et al. 2001; Lazarian, Pogosyan, \& Esquivel 2002) all have about the same overall power spectrum. 


\section{Stars Follow the Gas}

Stellar structures, such as clusters and flocculent spiral arms, have hierarchical geometries (Feitzinger \& Galinski 1987; Gomez et al. 1993; Elmegreen \& Elmegreen 2001; Zhang, Fall, \& Whitmore 2001) and power-law power spectra (Elmegreen, Elmegreen, \& Leitner 2003; Elmegreen et al. 2003) that are nearly identical to those of the gas. Star formation also has a duration that scales with the region size in the same way as the turbulent crossing time scales with size (Efremov \& Elmegreen 1998). These similarities between star formation and turbulent gas imply that star formation follows the gas to first order, i.e., that turbulence controls the star formation density, rate, and morphology. This control apparently extends to small scales too, perhaps down to individual binary stars (Larson 1995), as the protostars in clusters sometimes have their own hierarchical structure (Motte, André, \& Neri 1998; Testi et al. 2000). The large formation rates and high densities of embedded protostars also suggest that turbulence compresses the gas in which they form (Elmegreen \& Shadmehri 2003).

\section{Triggering}

Closer examination shows a second-order effect: a fairly high fraction of star formation is triggered inside pre-existing clouds by external pressures unrelated to the clouds and to the pressures of the current generation. These processes are revealed by the wind-swept appearance of many cluster-forming clouds (e.g., de Geus 1992; Bally et al. 1987) and by the proximity of cluster-forming cores to external HII regions (Yamaguchi et al. 1999; Walborn et al. 1999; Heydari et al. 2001; Yamaguchi et al. 2001a,b; Deharveng et al. 2003). Probably supersonic turbulence and entrainment in a multi-phase ISM produce the basic cloudy structure, and then pressure fluctuations in the environment trigger star formation in these structures (Elmegreen 2002). There would still be star formation without the triggers, but with a smaller rate per cloud because of the lower cloud densities, and a higher number of active clouds because of the more dispersed nature of the dense sub-regions. The influence of pressurized triggering on the star formation rate in a galaxy is not known, but the universal scaling of star formation rate with average column density (Kennicutt 1998) suggests that any direct influence is weak. Star formation is probably saturated to the maximum rate allowed in a compressibly turbulent medium (Elmegreen 2002).

\section{Size of Sample Effects}

The stochastic nature of turbulence is also reflected in the formation of star clusters, which show a random size-of-sample effect with regard to maximum mass. This appears in several ways: the most massive stars in a cluster increase with the cluster mass (Elmegreen 1983), the most massive clusters in a galaxy increase with the number of clusters (Whitmore 2003; Billett et al. 2002; Larsen 2002 ), and the most massive clusters in a logarithmic age interval increase with age (Hunter et al. 2003). In all cases, the slopes of these increases are determined exclusively by the mass function through the size of sample effect: bigger regions sample further out in the tail of the distribution and have more massive most- 
massive members. There is apparently no physical effect that has yet been found to determine the most massive member of a population. This is true even for individual stars (Massey \& Hunter 1998; Selman et al. 1999) although stellar radiation pressure and winds could limit the stellar mass once it gets large enough (Yorke \& Sonnhalter 2002; but see McKee \& Tan 2003).

Similarly, the ISM pressure should limit the cluster mass, considering that a cluster is recognized only if its density exceeds a certain value (depending on the sensitivity of the observation), and the density, mass and pressure are related by the virial theorem with a boundary condition. Nevertheless, this pressure limit for massive clusters has not been seen yet. It would appear as a drop-off at the upper end of the cluster mass function in a very large galaxy (sampling lots of clusters) with a low pressure (such as a giant low-surface brightness galaxy). Most galaxies have their sample-limiting mass comparable to or less than their pressure-limiting mass. Dwarf star-burst galaxies are an extreme example of this because they have very few clusters overall and yet some high pressure regions. Dwarf galaxies do indeed have an erratic presence of massive clusters, some of which may be related to galaxy interactions (Billett et al. 2002).

\section{Summary}

Most stars form in clusters (Carpenter 2000; Lada \& Lada 2003) and many of these clusters are close enough to high-pressure regions to look triggered. Triggering seems necessary because the dynamical pressures inside clusters are several orders of magnitude larger than the ambient interstellar pressure. The high pressure state of a cluster is an obvious remnant of its birth, but clues to the origin of the pressure are lost once the gas disperses and the stellar orbits mix. The primary distinction between the formation of standard "open clusters" and the mere aggregation of stars in a compressibly turbulent medium is probably this last step of triggering. HII regions did not compress gas to make Gould's Belt, but they did compress gas to make the Trapezium cluster in Orion.

The masses and positions of the clouds that are compressed into clusters seem to be the result of interstellar turbulence and shell formation. Turbulence structures the gas in two ways: by direct compression through random largescale flows, and by moving pre-existing clouds around passively. This duality of processes follows from the multi-phase nature of the ISM and from the presence of self-gravity. Combine these with pervasive pressure bursts from massive stars and the result is a mode of star formation dominated by dense clusters.

\section{References}

Bally, J., Langer, W.D., Stark, A.A., \& Wilson, R.W. 1987, ApJ, 312, L45

Bate, I.A., Bonnell, M.R., \& Bromm, V. 2003, MNRAS, 339, 577

Billett, O.H., Hunter, D.A., \& Elmegreen, B.G. 2002, AJ, 1231454

Carpenter, J.M. 2000, AJ, 120, 3139

de Geus, E.J. 1992, A\&A, 262, 258

Deharveng, L., Zavagno, A., Salas, L. et al.: 2003, A\&A, 399, 1135

Dickey J., McClure-Griffiths N., Stanimirovic S. et al: 2001 ApJ 561, 264 
Efremov, Y.N. 1995, AJ, 110, 2757

Efremov, Y.N., \& Elmegreen, B.G. 1998, MNRAS, 299, 588

Elmegreen, B.G. 1983, MNRAS, 203, 1011

Elmegreen, B.G. 1994, ApJ, 433, 39

Elmegreen, B.G. 2002, ApJ, 577, 206

Elmegreen, B.G. \& Elmegreen, D.M. 1987, ApJ, 320, 182

Elmegreen, B.G. \& Elmegreen, D.M. 2001, AJ, 121,1507

Elmegreen, B. G., Kim, S., \& Staveley-Smith, L. 2001, ApJ, 548, 749

Elmegreen, B.G. \& Shadmehri, M. 2003, MNRAS, 338, 817

Elmegreen, D.M., Elmegreen, B.G., \& Eberwein, K.S. 2002, ApJ, 564, 234

Elmegreen, B.G., Elmegreen, D.M., \& Leitner, S.N., 2003, ApJ, 590, 271

Elmegreen, B.G., Leitner, S.N., Elmegreen, D.M., \& Cuillandre, J.-C. 2003, ApJ, 593, 333

Feitzinger, J. V. \& Galinski, T. 1987, A\&A, 179, 249

Goldman, I. 2000, ApJ, 541, 701

Gomez, M., Hartmann, L., Kenyon, S. J., \& Hewett, R. 1993, AJ, 105, 1927

Grabelsky, D.A., Cohen, R.S., May, J. et al: 1987, ApJ, 315, 122

Heydari, M., Charmandaris, V., Deharveng, L. et al.: 2001, A\&A, 372, 495

Hunter, D., Elmegreen, B.G., Dupuy, T., Mortonson, M. 2003, AJ, 126, 1836

Kennicutt, R.C. 1998, ApJ, 498, 541

Kim, S., Dopita, M.A., Staveley-Smith, L., Bessell, M.S. 1999, AJ, 118, 2797

Kim, W.-T. \& Ostriker, E.C. 2002, ApJ, 570, 132

Lada, C.J. \& Lada, E.A. 2003, ARAA, 41, 57

Larsen, S. S. 2002, AJ, 124, 1393

Larson, R.B. 1995, MNRAS, 272, 213

Lazarian, A., Pogosyan, D., \& Esquivel, A. 2002, A.SP Conf. Proc. 276, Seeing Through the Dust, p. 182

Massey, P. \& Hunter, D.A. 1998, ApJ, 493, 180

McKee, C.F. \& Tan, J.C. 2003, ApJ, 585, 850

Motte, F., Andre, P. \& Neri, R. 1998, A\&A, 336, 150

Rand, R.J. 1993, ApJ, 410, 68

Selman, F., Melnick, J., Bosch, G., \& Terlevich, R. 1999, A\&A, 347, 532

Stanimirovic S., Staveley-Smith L., Dickey J. et al: 1999 MNRAS 302, 417

Stützki, J., Bensch, F., Heithausen, A. et al.: 1998, A\&A, 336, 697

Testi, L. et al. 2000, ApJ, 540, L53

Walborn, N.R., Barbá, R.H., Brandner, W. et al.: 1999, AJ, 117, 225

Whitmore, B. C. 2003, in STScI Symp. 14, A Decade of HST Science, ed. M. Livio, K. Noll, \& M. Stiavelli (Baltimore: STScI), 14, 153

Yamaguchi, R., Saito, H., Mizuno, N. et al.: 1999, PASJ, 51, 791

Yamaguchi, R., Mizuno, N., Onishi, T. et al.: 2001a, ApJ, 553, 185

Yamaguchi, R., Mizuno, N., Onishi, T. et al.: 2001b, PASJ, 53, 959

Yorke, H.W. \& Sonnhalter, C. 2002, ApJ, 569, 846

Zhang, Q., Fall, S. M., \& Whitmore, B. C. 2001, ApJ, 561, 727 